Recepción: 13 / 04 / 2018

Aceptación: 25 / 06 / 2018

Publicación: 01 / 08 / 2018

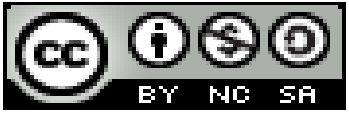

Ciencias de la salud Artículo de investigación

\title{
Automedicación y los riesgos en la salud de la población adulta
}

\section{Self-Medication and the health Risks of adult population}

\section{Auto-medicação e os riscos para a saúde da população adulta}

\author{
Mercedes T. Alcázar-Pichucho ${ }^{\mathrm{I}}$ \\ taniameralcazar@hotmail.es \\ Roberth O. Zambrano-Santos II \\ rzambranosantos@yahoo.es \\ Virginia E. Pincay-Pin III \\ pincaypinvirginia@yahoo.com
}

\section{Correspondencia: taniameralcazar@hotmail.es}

\footnotetext{
${ }^{\text {I }}$ Magister en gerencia en salud para el desarrollo local, Licenciada en ciencias de la enfermería, Docente de la Universidad Estatal del Sur de Manabí, Jipijapa, Ecuador.
}
II Magister en desarrollo educativo, Magister en gerencia de proyectos educativos y sociales, Doctor en ciencias de la educación especialidad investigación socio educativa, Licenciado en ciencias de la educación especialización historia y geografía, Docente de la Universidad Estatal del Sur de Manabí, Jipijapa, Ecuador.

III Magister en gerencia en salud para el desarrollo local, Licenciada en ciencias de la enfermería, Docente de la Universidad Estatal del Sur de Manabí, Jipijapa, Ecuador. 


\title{
Resumen
}

La automedicación implica riesgos en la salud por el desconocimiento y el uso inadecuado de los medicamentos, genera complicaciones a largo o corto plazo, como; resistencias de medicamentos, dependencias, adicción, enmascaramiento de enfermedades, el problema científico es la deficiencia de conocimiento de los riesgos de salud por la automedicación, ya que son muchas las personas que se automedican sin llegar a conocer las dificultades que ocasiona, como objeto de la investigación la automedicación y la relación que existe con los riesgos de salud, prevaleciendo de manera significativa las características de esta problemática y sus consecuencias en el campo de la salud pública, ya que es un problema sanitario a nivel mundial. El objetivo fue determinar los riesgos de la automedicación en la población adulta de 30 -45 años de edad en la Parroquia Parrales y Guales del Cantón Jipijapa. Para la elaboración de esta investigación se empleó métodos teóricos de inducción - deducción y método empírico mediante técnicas de encuestas y entrevistas, se obtuvo como resultado que la población adulta se auto medican, siendo los analgésicos el fármaco más usado, los cuales son obtenidos en farmacias por decisión propia, demostrándonos que los habitantes están en riesgo de presentar problemas de salud.

Palabras clave: medicamentos; complicaciones; resistencia; intoxicaciones.

\begin{abstract}
Self-medication entails health risks due to lack of knowledge and inadequate use of drugs, generates long-term or short-term complications, as; Resistance of drugs, addictions, addiction, masking of diseases, so the scientific problem is defined as the deficiency of knowledge of health risks by self-medication, since many people self-medicate without knowing the difficulties That this can cause, as the object of the research, self-medication and the relationship that exists with health risks, the characteristics of this problem and its consequences in the field of public health prevailing in a significant way, since it is a health problem worldwide. The objective was to determine the risks of self-medication in the adult population of 30-45 years of age in Parish Parrales and Goals of the Canton Jipijapa. For the elaboration of this research was used theoretical methods of induction - deduction and empirical method by techniques of surveys and interviews, was obtained as a result that the adult population self - medicate, with analgesics
\end{abstract}


being the drug most used, which are obtained in pharmacies by their own decision, demonstrating that the inhabitants are at risk of presenting health problems.

Keywords: danger; drugs; complications; resistance; adverse reactions; poisonings.

\section{Resumo}

A automedicação envolve riscos à saúde devido à ignorância e uso inadequado de medicamentos, gera complicações a longo ou curto prazo, como; Resistências de drogas, dependência, dependência, mascarando a doença, o problema científico é a deficiência de conhecimento dos riscos para a saúde da automedicação, como muitas pessoas que se auto-medicar sem conhecer as dificuldades causadas como um objeto de a pesquisa da automedicação e a relação que existe com os riscos à saúde, prevalecendo significativamente as características desse problema e suas consequências no campo da saúde pública, por se tratar de um problema de saúde global. O objetivo foi determinar os riscos da automedicação na população adulta de 30 a 45 anos na paróquia de Parrales y Guales, no cantão de Jipijapa. Para o desenvolvimento desta pesquisa métodos de indução teóricos foi usado - dedução e método empírico usando técnicas de pesquisas e entrevistas, foi obtida como resultado que a população adulta auto-medicar, os analgésicos drogas mais usadas, que são obtidos em farmácias por decisão própria, demonstrando que os moradores correm o risco de apresentar problemas de saúde.

Palavras chave: medicamentos; complicações; resistência; envenenamento.

\section{Introducción}

La automedicación es la utilización de medicamentos por decisión propia sin ninguna intervención por parte del médico, ni en el diagnóstico de la enfermedad, ni en la prescripción o supervisión del tratamiento. La automedicación en la población adulta se ha convertido en una conducta que implica tomar medicamentos sin receta médica, por voluntad propia, o prescripciones médicas anteriores, sin percatarse que esta puede ocasionar daños irreparables en la salud, es un hecho habitual y creciente que implica un uso indiscriminado de medicamentos. La automedicación es un tipo de conducta que implica tomar medicamentos según la propia iniciativa del paciente, por consejo del farmacéutico o de cualquier persona no médica (Morillo \& Valencia, 2010). La automedicación es el consumo de medicamentos sin receta médica, esta 
práctica es un problema de salud pública a nivel mundial y es la causante principal de la resistencia antibiótica (Cordero, Cardoso, \& Crespo, 2014).

Con lo expuesto la automedicación es una acción que acarrea grandes consecuencias, provoca complicaciones y enmascaramiento de enfermedades, resistencias de medicamentos, padecimientos de reacciones adversas, alergias e intoxicaciones, y dependencia de fármacos que puedan poner en riesgo la salud y causar la muerte. Se sabe que muchas personas se automedican, pero se desconoce la situación real de la automedicación.

La Organización Mundial de la Salud (OMS) (2010) define la automedicación como el uso de los medicamentos por parte de las personas con el propósito de tratar enfermedades o síntomas que ellos mismos puedan identificar. Se refiere fundamentalmente a los medicamentos de venta libre. Y la auto prescripción como el uso indiscriminado de fármacos sin indicación ni supervisión facultativa.

La automedicación es normal entre individuos que lo ejecutan, por simple referencia de casos parecidos y semejantes a otras enfermedades, y cada día se incrementa su práctica en las naciones, lo cual llama la atención de forma preocupante, por los efectos perjudiciales en la salud de las personas (Ruiz \& Pérez, 2014).

La mayoría de la población adulta se automedica, sin prever que todo fármaco por más sencillo que se muestre cuando actúa en el organismo humano puede presentar reacciones adversas y consecuencias. El consumo de medicamentos de cualquier grupo sin la prescripción médica depende de muchos factores: la no exigencia de una receta médica para dispensar el medicamento, factores económicos, la disponibilidad del fármaco, poca disponibilidad de tiempo para acudir a los servicios de salud, mala atención por parte de las entidades de salud, pérdida de credibilidad de los médicos, las conductas que asumen las personas frente a las enfermedades.

La automedicación se ha convertido en un hábito frecuente por la necesidad de las personas ante alguna sintomatología y por este motivo se la define como "el consumo de medicamentos sin la intervención de los profesionales de la salud, porque se conoce que existe un gran porcentaje de la población que se auto médica, y suele ser víctima de reacciones no deseadas" (Domínguez, 2013).

La intervención del profesional de salud en la aparición de enfermedades es muy importante ya que mediante una valoración puede determinar un diagnostico que puede salvaguardar la vida del 
paciente, sin embargo, hay personas que prefieren no ser atendidos y tomar medicamentos por su propia cuenta.

"Estudios realizados en Brasil manifiestan que las personas prefieren automedicarse por las siguientes razones: comodidad (no tener que pedir cita), no hacer colas largas, no perder tiempo, economía (se ahorra una consulta), le resuelve su situación más rápido y se siente responsable con su salud. El $88 \%$ de los entrevistados conocen los factores de riesgo asociados a los medicamentos y, a pesar de ello, los utilizan sin una necesidad real. Los restantes $12 \%$ no saben o no responden" (Tobón, 2002, p. 8,9).

La mayor parte de la población desconoce los riesgos que ocasiona la toma de medicamentos y está consciente de la problemática sin embargo es conveniente que las instituciones de salud pongan mayor énfasis en esta situación ya que también depende de ellos que los pacientes acuden de forma responsable a los controles médicos y además que los señores farmacéuticos expendan sus medicamentos con receta médica.

De igual manera Ecuador no está aparte de este problema, así muestra la universidad de Cuenca en la investigación sobre la automedicación y factores asociados en estudiantes del Colegio Manuel Garaicoa de Calderon, Cuenca, de la cual la prevalencia de automedicación en un 51\%; los medicamentos de mayor uso fueron los analgésicos y antiinflamatorios con el 84,3\%; el principal motivo fue presentar sintomatología leve (68,6\%), la fuente de información fue la recomendación de un amigo o familiar (47,8\%; en el 69,8\% la farmacia fue el sitio de adquisición de los medicamentos; el 28,3\% no mejoro su sintomatología; el 43,4\% de la población desconoce los riesgos de la automedicación (Castro, Cobos, \& Lazzo, 2013,p. 38-44).

Uno de los síntomas más comunes de la automedicación es el dolor de cabeza, llevando a ingerir de manera excesiva los analgésicos para aliviar este síntoma, sin importar que el diagnostico medico es importante para determinar si es migraña o cefalea de tensión o puede ser un síntoma que acompaña otra patología, empeorando así su situación clínica, o llegando a la complicación de enfermedades. Dado que existen estudios relativos a este problema, se evidencia que los analgésicos son muy utilizados por su poder calmante o paliativo sin presagiar los efectos y consecuencias que estos pueden llegar a ocasionar en el organismo. 
Alvarado \& Vargas (2013, p.81) refieren que "el uso regular y frecuente de analgésicos como aspirina, codeína, dextropropoxifeno y paracetamol, particularmente cuando se toman juntos para trata cefaleas no específicas, puede causar cefalea crónica como reacción paradójica". Así también nos refiere que: La enfermedad hepática se da por el abuso crónico de paracetamol como analgésico logrando resultar muy perjudicial para la salud, ya que produce insuficiencia hepática que muchas veces resulta mortal.

La ingesta abundante de analgésicos, logra problemas o daños especialmente el hígado y riñón, como es la hepatoxicidad, nefrotoxicidad, insuficiencia renal, entre otros, muchos de los cuales pueden ser severos cuando actúan en el sistema nervioso, producen somnolencias, mareos, confusión, letargo, etc., muy peligrosos para adultos mayores y niños, porque al no poder expresar lo que sienten corren riesgos de caídas, y más aún en sobredosis independientemente de la edad del paciente puede causar la muerte.

Para Acosta (2013, p.15) "el paciente no conoce la dosis conveniente para su condición, y puede que se cause grandes estragos al tomar mayor cantidad de la medicina, mientras que, si ingiere la cantidad adecuada, el medicamento hará lo suyo y curará la dolencia", porque si ingiere menor dosis el organismo no le aprovechará, y si es mayor tampoco y ambos casos en vez de curar traerá más complicaciones a la enfermedad.

El desconocimiento es la principal causa de cometer errores y hasta provocar complicaciones en el tratamiento de una enfermedad, los medicamentos cumplen su función si son utilizados de acuerdo a la dosificación que indique el profesional de la salud, pero al no intervenir el profesional la equivocación o no la correcta dosis del medicamento genera complicaciones graves.

El paciente se auto médica no solo con los medicamentos de venta libre sino también con aquellos que se venden bajo prescripción médica. Esta conducta induce al uso irracional de los medicamentos más usados como analgésicos, antibióticos, antihistamínicos, antiácidos, antiinflamatorios, sin embargo, los peligros son múltiples. Por ejemplo, se abusa de fármacos para obtener efectos que nada tienen que ver con sus propiedades. El resultado es el contrario al deseado puede causar complicaciones, enfermedades a lo largo del tiempo incluso su acción cuando realmente se los necesite (Lazcano, Parra, \& Umeres, 2014). 
Este tipo de prácticas es un problema serio, que trasciende fronteras, culturas, nivel académico, estrato socioeconómico además de ser responsable de morbilidad y en casos mortalidad en todo el mundo. Es, por tanto, que instancias internacionales como (OMS), han tenido la iniciativa de promover la educación integral en cuanto al auto cuidado, para así disminuir las prácticas irresponsables de automedicación, entre otros objetivos (Amaya, 2010).

En este sentido, forma parte de las actividades del autocuidado de la salud, al igual que el cuidado de la higiene, la nutrición, el estilo de vida, y la influencia de factores socioeconómicos y ambientales, dentro de este marco quedan incluidos los conceptos de automedicación y de automedicación responsable. En la automedicación responsable, el usuario trata sus enfermedades o síntomas con medicamentos que han sido aprobados, están disponibles para la venta sin prescripción y que son seguros y eficaces cuando se usan en las condiciones establecidas.

El presente trabajo de investigación tiene como problema científico, las deficiencias de conocimientos de los riesgos de salud por la automedicación en la población adulta, ya que muchos estudios manifiestan que no existe una verdadera concientización de parte de las personas adultas, que se auto medican sin prever los riesgos a los que están expuestos.

Se define como objeto de estudio a la automedicación en la población adulta y la relación que existe con los riesgos en la salud, priorizando de manera significativa los factores que inciden ante esta problemática y sus consecuencias que en muchas ocasiones pueden llegar hasta la muerte, ya que los medicamentos tienen diferentes interacciones.

Este trabajo está inmerso en el campo de la salud pública ya que para esta constituye un grave problema sanitario que afecta a la comunidad en general pero que aun así se puede prevenir. La salud pública enmarcada a mejorar la salud de la población y encaminada a proteger a la población más vulnerable de aquellos factores que pueden generar enfermedad, promueve la prevención primaria en salud necesaria para concientizar a la comunidad a mejorar su nivel de vida.

El objetivo general de este trabajo es determinar los riesgos de la automedicación en la población adulta de 30 - 45 años de edad.

Importancia del problema 
La automedicación y los riesgos en la salud de la población adulta, es una problemática que amerita una atención urgente por parte de toda la población, responsabilidad que también es asumida por los expendedores de medicamentos, quienes deben abstenerse de medicar a todos quienes se acercan a las farmacias y que no cuentan con una prescripción médica. Los beneficiarios de este proyecto fueron los habitantes del cantón Jipijapa, Ecuador.

\section{Metodología}

La investigación es de tipo cuantitativa y cualitativa, la población de estudio fue de 970 adultos de 30 a 45 años de edad, la técnica para la selección de la muestra fue a través de una muestra probabilística por medio de encuestas diseñada de tal manera que los datos puedan ser generalizados, realizando una asociación entre ambas variables cuyos resultados servirán de información para tomar decisiones. Para analizar los datos de manera objetiva, segura, confiable y precisa, se utilizaron los métodos de Inducción-deducción y el empírico mediante la observación, se obtuvieron conclusiones de lógica formal permitiéndonos conocer la realidad, analizar la problemática y hacer relaciones esenciales entre automedicación y el riesgo de salud en la población adulta.

En este proceso se recolectaron 197 encuestas ejecutadas en los habitantes de la Parroquia Parrales y Guale, perteneciente al Cantón Jipijapa. Al analizar la información obtenida de esta cifra de encuestados, y para su mayor compresión en el sondeo se agruparon en tres rangos de edades de 30 - 35 años, en de 36 a 40 años, y en las edades de 41 a 45 años. También se reveló que el $56 \%$ son del sexo femenino y el $44 \%$ son del género masculino.

\section{Resultados}

Figura 1. Lugar al que se acude ante una molestia de salud

\begin{tabular}{lcc}
\hline \multicolumn{1}{c}{ ALTERNATIVAS } & FRECUENCIA & PORCENTAJE \\
\hline $\begin{array}{l}\text { MSP (Hospitales, centro } \\
\text { de salud) }\end{array}$ & 27 & 14 \\
$\begin{array}{l}\text { Privado (Clínicas, } \\
\text { hospitales particulares) }\end{array}$ & 10 & 5 \\
Farmacias & 138 & 70 \\
Otros & 22 & 11 \\
\hline
\end{tabular}


TOTAL

197

100

\section{Fuente: Elaboración propia.}

El presente resultado demuestra que la mayor parte de adultos acuden en primera instancia a la farmacia cuando presenta algún malestar, sin darse cuenta que puede haber una interacción medicamentosa entre fármacos obtenidos en la farmacia y fármacos de cumplimiento terapéutico. Es por esto que se indago la preferencia en cuanto al lugar que elige al momento que se encuentra delicado de salud, consiguiendo identificar que la población encuestada acude a las farmacias ante una molestia de salud esto lo demuestra el 70\% de las encuestas aplicadas, mientras que el $14 \%$ acude a las instituciones que pertenecen al Ministerio de Salud Pública, y en muy poco porcentaje a las instituciones privadas. Esto determina que la población confía plenamente en la adquisición de medicamentos en las farmacias por determinación propia para aliviar su enfermedad.

Figura 2. Consumo de medicamentos por iniciativa propia en los últimos seis meses

\begin{tabular}{lll} 
ALTERNATIVAS & FRECUENCIA & PORCENTAJE \\
\hline $\mathrm{Si}$ & 160 & 81 \\
$\mathrm{No}$ & 37 & 19 \\
\hline TOTAL & $\mathbf{1 9 7}$ & $\mathbf{1 0 0}$ \\
\hline
\end{tabular}

Fuente: Elaboración propia.

La mayor parte de adultos mayores no tienen conocimiento sobre el efecto de los medicamentos que consumen día a día esto nos refleja una mala educación hacia el usuario, por lo que el consumo de medicamentos se lo realiza sin prescripción médica, sin la intervención de un profesional que valore el estado de salud del paciente y más bien es por iniciativa propia que adquieren diferentes fármacos que a la larga resulta perjudicial en la salud de las personas.

Figura 3. Motivo por los que las personas prefieren automedicarse

\begin{tabular}{lcc}
\hline ALTERNATIVAS & FRECUENCIA & PORCENTAJE \\
\hline Falta de recursos & 6 & 4 \\
económicos & 28 & 18 \\
No dispone de tiempo & 23 & 14 \\
Facilidad para comprar & 19 & 12 \\
(farmacias) & & \\
Falta de profesionales de & & \\
442 & & \\
\hline
\end{tabular}


la salud

Por recomendaciones de

24

15

otra persona

Decisión propia

60

38

TOTAL

160

100

Fuente: Elaboración propia.

La automedicación es una práctica muy común en todo el mundo, siendo los factores económicos, sociales y culturales las principales causas que favorecen que se presente habitualmente, el resultado de la encuesta demuestra que la mayor parte de adultos mayores se automedican por decisión propia sin concientizar los problemas de salud que ocasiona la automedicación, otros refieren que es por falta de tiempo para acudir al médico lo que ocasiona que aumente proporcionalmente los casos de personas que se automedican.

Figura 4. Síntomas por los que la población se ha automedicado.

\begin{tabular}{lcc}
\hline ALTERNATIVAS & FRECUENCIA & PORCENTAJE \\
\hline Gripe & 25 & 16 \\
Dolor de cabeza & 43 & 27 \\
Dolor de garganta & 17 & 11 \\
Dolores musculares & 51 & 32 \\
Diarrea & 1 & 1 \\
Fiebre & 11 & 7 \\
Ardor y dolor estomacal & 7 & 4 \\
Cólicos menstruales & 4 & 3 \\
Afecciones a la piel. & 1 & 1 \\
\hline TOTAL & $\mathbf{1 6 0}$ & $\mathbf{1 0 0}$ \\
\hline
\end{tabular}

Fuente: Elaboración propia.

Las personas, se consideran lo suficientemente capacitados para distinguir los síntomas leves y, por lo tanto, para automedicarse sin necesidad de consejo médico ni farmacéutico, los dolores musculares y el dolor de cabeza son los síntomas más comunes por lo que la población se 
automedica, lo que la población desconoce es que estos síntomas son acompañantes de una enfermedad y es aquí donde se produce el mayor consumo de analgésicos para aliviar la aparición de estos malestares.

Figura 5. Reacciones negativas presentadas por ingerir medicamentos sin prescripción médica.

\begin{tabular}{|c|c|c|}
\hline ALTERNATIVAS & FRECUENCIA & PORCENTAJE \\
\hline Intoxicación & 12 & 6 \\
\hline Alergia & 14 & 7 \\
\hline $\begin{array}{l}\text { Resistencia a los } \\
\text { antibióticos }\end{array}$ & 6 & 3 \\
\hline $\begin{array}{l}\text { Complicaciones en su } \\
\text { enfermedad }\end{array}$ & 24 & 12 \\
\hline Dependencia o adicción & 31 & 16 \\
\hline $\begin{array}{l}* \text { *SUBTOTAL }(\Sigma \\
\text { Reacciones negativas) }\end{array}$ & 87 & 44 \\
\hline Ninguna & 110 & 56 \\
\hline TOTAL & 197 & 100 \\
\hline
\end{tabular}

Fuente: Elaboración propia.

Consumir medicamentos sin prescripción médica puede traer consecuencias leves o severas sobre el organismo de quienes lo consumen Elegir medicamentos sin prescripción médica ocasiona la aparición de reacciones negativas que afectan nuestro organismo, ya sea a corto o largo plazo porque el consumo de medicamentos no está direccionado por un médico y en la toma puede haber errores que pueden dar lugar a reacciones que pueden llegar hasta la muerte esto lo refleja que el $44 \%$ de los encuestados presentaron reacciones como intoxicación, alergias, resistencia a antibióticos, complicaciones en su enfermedad, lo que demuestra los inconvenientes que se presentan por la ingesta de medicamentos sin prescripción médica.

\section{Discusión}

Por el termino automedicación, entendemos la adquisición de fármacos sin receta médica, provenientes del entorno cercano, bien por la recuperación de medicamentos utilizados en otras 
ocasiones, o por la prolongación, interrupción o modificación de las dosis indicadas en un tratamiento ya prescrito.

En México los temas que abordan sobre el uso racional de medicamentos distinguen la regulación entre aquellos medicamentos que requieren para su venta receta médica, y aquellos que no la requieren, teniendo un margen de riesgo/ beneficio que permiten su utilización sin supervisión médica para el alivio de una serie de síntomas (dolor de cabeza, fiebre, acidez estomacal, etc.). En este sentido, la automedicación es una forma de autocuidado de la salud y no debe catalogarse como una práctica peligrosa (Dreser \& Leyva, 2009).

Un estudio realizado en Barranquilla (Colombia) demuestra que los antibióticos son medicamentos importantes, pero se los prescribe en exceso, y además hay quienes se los automedican, haciendo un uso abusivo de ellos para tratar trastornos como diarreas, resfrió y tos. Cuando los antibióticos se usan con demasiada frecuencia y en dosis inferiores a las recomendadas, las bacterias se vuelven resistentes a ellos.

Los adultos aparecen como el grupo de mayor riesgo con relación a las reacciones adversas debido a una disminución progresiva del funcionamiento de órganos y sistemas, que provoca cambios generales y efectos en cadena dentro del propio organismo. La edad influye de forma general en los receptores farmacológicos. Si unimos la edad y la enfermedad el organismo da una respuesta a los fármacos y esto impide detectar las reacciones adversas a medicamentos.

Ahora en la actualidad más los grandes descubrimientos de la medicina, en los que existen fármacos que dan solución ante cualquier sintomatología, hacen de esta práctica necesaria y oportuna para el alivio o cura de cualquier problema de salud, permitiendo así a las personas y por voluntad propia acceder a la utilización de fármacos o medicamentos para su tratamiento sin receta médica, poniendo en riesgo su salud, ya que sin un conocimiento previo de enfermedad y medicamento a utilizar puede ocasionar graves consecuencias o perjuicios en la salud.

El presente estudio demuestra que los riesgos relacionados a la automedicación son muchos, pero las personas no son conscientes de que estos pueden ocasionar graves problemas en la salud como: enmascaramiento y agravamiento de la enfermedad, efectos adversos (alergias, náuseas, cefaleas, mareos, diarreas, gastritis), disminución de la efectividad, Resistencia a los antibióticos, intoxicación y hasta la muerte. 
Mediante pacientes adecuadamente informados conseguiremos disminuir tanto la presencia de automedicación como el incumplimiento terapéutico, es por esto que el presente estudio considera necesario promover la información con el fin de prevenir posibles efectos adversos relacionados con la automedicación.

\section{Conclusiones}

La investigación llevada a cabo nos ha permitido realizar un profundo análisis de la situación de los riesgos de salud referente a la automedicación por ello se concluye con lo siguiente:

La práctica de automedicarse es un mal que aqueja la gran parte de la sociedad encuestada y que es coincidida por especialistas en la salud que aducen que esto va en aumento por la falta de educación, con los resultados se evidencian que los habitantes prefieren acudir a las farmacias que a las instalaciones de los hospitales, haciendo referencia que no existe personal de salud suficiente para suplir las necesidades de los usuarios, y por ellos deciden administrarse fármacos sin prescripción médica. También se demuestra que los habitantes aun no creen en desarrollo de la salud pública.

Otras de las preocupaciones latentes es que no existe medida urgente donde se prohíba la venta de muchos medicamentos sin recetas médicas, porque hay facilidad para su compra; y los fármacos más recurrentes en su adquisición son los analgésicos, Se expone con nuestra investigación que gran parte de los individuos presenta reacciones adversas, y con todo lo manifestado con anterioridad, ellos indican que volverían automedicarse. 


\section{Referencias Bibliográficas}

Acosta, A. (2013). Estudio sobre los factores que inciden en el habito de la automedicación.

UNAD. [en línea], Disponibles en: https://webcache.googleusercontent.com/search?q=cache:AN_K0S8eOwsJ:https://stadium.unad.e du.co/preview/UNAD.php\%3Furl\%3D/bitstream/10596/1754/1/1027960966.pdf +\&cd=1\&hl=es$419 \& \mathrm{ct}=\mathrm{clnk} \& \mathrm{gl}=\mathrm{ve} \& \mathrm{client}=$ firefox $-\mathrm{b}-\mathrm{ab}$.

Alvarado, M., \& Vargas, J. (2017). Prevalencia y factores asociados a la automedicación en estudiantes de Medicina. Revista de la Facultad de Medicina Humana. 17(4):80-85, [en línea]., Disponibles en: http://dspace.unapiquitos.edu.pe/bitstream/unapiquitos/188/1/1. Obtenido de Características de la Automedicación.

Amaya, R. (2010). Caracterización de la automedicación de la población mayor de 18 años residente en el municipio de San Ignacio en Chalatenango [en línea], Disponibles en: http://hdl.handle.net/10972/967. Obtenido de REDICCES

Castro, R., Cobos, G., \& Lazzo, R. (2013) Automedicación y factores asociados en estudiantes del Colegio Manuela Garaícoa de Calderón, Cuenca 2012, [en línea], Disponibles en: http://dspace.ucuenca.edu.ec/bitstream/123456789/3691/1/MED168.pdf.

Cordero, V., Cardoso, L., \& Crespo, A. (2014). Prevalencia de automedicación con antibióticos y factores asociados, en adultos mayores en el área urbana del Cantón cuenca, 2013-2014. [en línea], Disponibles en: http://dspace.ucuenca.edu.ec/bitstream/123456789/21035/1/TESIS.pdf Domínguez, G.I; Martínez, L.M- y Rodríguez, M. (2013). Características del consumo de medicamentos de venta libre en una población de adultos de la ciudad de Medellín (Colombia). Revista Salud Uninorte. Barranquilla (Col.); 29 (3): 360-367, [en línea], Disponibles en: http://www.scielo.org.co/pdf/sun/v29n3/v29n3a02.pdf.

Dreser, A., \& Leyva, R. (2009). Retos y oportunidades para el desarrollo de la política farmacéutica nacional en México. Revista Salud Pública de México. Vvol. 55, No. 3, mayo-junio, 2013, pp. 329-336. [en línea], Disponible en: http://www.redalyc.org/pdf/106/10628332011.pdf. Lazcano, L., Parra, E., \& Umeres, L. (2014). Causas que inducen la automedicación en estudiantes de primer y quinto curso de la Facultad de Medicina. ECORFAM. [en línea], Disponible en: https://www.ecorfan.org/series/administracion/Topicos_Selectos_de_Administracion_4.pdf 
Morillo, M., \& Valencia, V. (2010). Factores relacionados con la automedicación en los estudiantes de Enfermería. [en línea], Disponible en: http://repositorio.utn.edu.ec/bitstream/123456789/696/1/06\%20ENF\%20427\%20ART\%C3\%8D CULO\%20CIENT\%C3\%8DFICO.pdf

Organización Mundial de la Salud (OMS). (2010). Estrategia Mundial de la OMS para contener la resistencia a los antimicrobianos. [en línea], Disponible en: http://www.antibioticos.msssi.gob.es/PDF/resist_OMS_estrategia_mundial_contra_resistencias.p df.

Ruiz, A., \& Pérez, A. (2014). Automedicación y términos relacionados: una reflexión conceptual. Revista Ciencias de la Salud. Vol. 9, No. 1. [en línea], Disponible en: https://revistas.urosario.edu.co/index.php/revsalud/article/view/1551.

Tobon, M. (2002). Estudio sobre automedicación. Revista IATREIA. Vol 15. No. 4. Diciembre. [en línea], Disponible en: http://www.scielo.org.co/pdf/iat/v15n4/v15n4a3.pdf. 